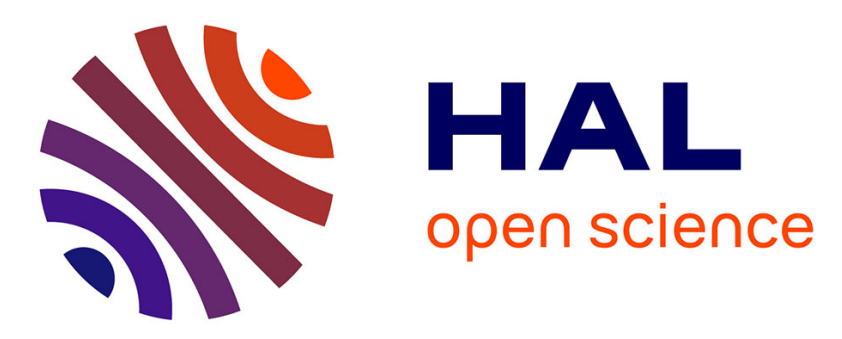

\title{
Inferring Information Across Scales in Acquired Complex Signals
}

\author{
Suman Kumar Maji, Oriol Pont, Hussein Yahia, Joel Sudre
}

\section{To cite this version:}

Suman Kumar Maji, Oriol Pont, Hussein Yahia, Joel Sudre. Inferring Information Across Scales in Acquired Complex Signals. European Conference on Complex Systems 2012, The Complex Systems Society, Sep 2012, Brussels, Belgium. pp.209-226, 10.1007/978-3-319-00395-5_31 . hal-00872371

\section{HAL Id: hal-00872371 \\ https://hal.inria.fr/hal-00872371}

Submitted on 11 Oct 2013

HAL is a multi-disciplinary open access archive for the deposit and dissemination of scientific research documents, whether they are published or not. The documents may come from teaching and research institutions in France or abroad, or from public or private research centers.
L'archive ouverte pluridisciplinaire HAL, est destinée au dépôt et à la diffusion de documents scientifiques de niveau recherche, publiés ou non, émanant des établissements d'enseignement et de recherche français ou étrangers, des laboratoires publics ou privés. 


\title{
Inferring Information across Scales in Acquired Complex Signals
}

\author{
Suman Kumar Maji ${ }^{1}$, Oriol Pont ${ }^{1}$, Hussein Yahia ${ }^{1}$ and Joel Sudre ${ }^{2}$ \\ 1 INRIA Bordeaux Sud-Ouest, Geostat team \\ suman-kumar.maji, oriol.pont, hussein.yahia@inria.fr \\ 2 LEGOS, UMR CNRS 5556, Dynbio Team \\ joel.sudre@legos.obs-mip.fr
}

\begin{abstract}
Transmission of information across the scales of a complex signal has some interesting potential, notably in the derivation of subpixel information, cross-scale inference and data fusion. It follows the structure of complex signals themselves, when they are considered as acquisitions of complex systems. In this work we contemplate the problem of cross-scale information inference through the determination of appropriate multiscale decomposition. Our goal is to derive a generic methodology that can be applied to propagate information across the scales in a wide variety of complex signals. Consequently, we first focus on the determination of appropriate multiscale characteristics, and we show that singularity exponents computed in microcanonical formulations are much better candidates for the characterization of transitions in complex signals : they outperform the classical «linear filtering» approach of the state-of-the-art edge detectors (for the case of $2 \mathrm{D}$ signals). This is a fundamental topic as edges are usually considered as important multiscale features in an image. The comparison is done within the formalism of reconstructible systems. Critical exponents, naturally associated to phase transitions and used in complex systems methods in the framework of criticality are key notions in Statistical Physics that can lead to the complete determination of the geometrical cascade properties in complex signals. We study optimal multiresolution analysis associated to critical exponents through the concept of «optimal wavelet». We demonstrate the usefulness of multiresolution analysis associated to critical exponents in two decisive examples : the reconstruction of perturbated optical phase in Adaptive Optics (AO) and the generation of high resolution ocean dynamics from low resolution altimetry data.
\end{abstract}

\section{Introduction}

Most real-world signals are complex signals, usually difficult to describe but possessing a high degree of redundancy [6]. In particular, in the case of Fully Developed Turbulence (FDT), there is a relation between the spectrum of singularity exponents associated to structure functions and the existence of a multiscale hierarchy [11]. Turbulent flows, although chaotic in nature, possess a 
complex arrangement of geometrical structures related to the cascading properties of physical variables [5]. The same type of conclusion can be inferred from multiscale analysis of most natural complex signals [12]. As a consequence, the paradigm of understanding natural signals as acquisitions of complex systems with unknown phase space is a useful one [14]. The properties of physical cascading variables reflect the transfer of energy, or more generally information, taking place from larger scales to smaller ones. Recent developments in microcanonical framework for the computation of singularity exponents and the derivation of singularity spectra have lead to a sensible improvement in the numerical techniques for the determination of multiscale characteristics of real signals $[1,2]$. Experimental analysis on different real world signals, ranging from stock market time series to atmospheric perturbated optical path shows that these systems are not only found to be multiscale, but their singularity spectra are also coincident. Consequently, the precise numerical computation of geometrically localized singularity exponents in single acquisitions of complex systems, without the averages taken on grand ensembles, unveils the determination of their universality class [1]. The statistical characteristics of information in these signals can be described from the localisation and precise value of singularity exponents. As a consequence, it should be possible to transfer across the scales extra physical information from lower scale to higher resolution, a procedure which unveils considerable enhancements of high resolution mapping of natural phenomena.

In this paper, we demonstrate that microcanonical formulations for understanding and evaluating the mechanisms that govern the evolution of dynamical systems lead to accurate inference schemes across the scales in complex signals. We show that the singularity exponents can be used in multiresolution analysis for accurate inference of information across the scales. The profound reason for this fact comes from the observation that geometrically localized singularity exponents encode transitions in complex signals in a much more accurate manner than done with linear filtering processing techniques [23,25], as will be demonstrated in this work, in particular in the case of 2D images and the accurate determination of edges (which are typically multiscale characteristics on an image). Consequently, we study the notion of optimal wavelet for inferring information across the scales. Our fundamental contribution in this work is to show that multiresolution analysis associated to geometrically localized singularity exponents is a very good candidate for inferring information across the scales in complex signals. We take two specific examples: the reconstruction of the optical phase shift perturbated by atmospheric turbulence (Adaptive Optics) and the high resolution mapping of ocean dynamics using sea surface temperature maps. In the first example, we derive a radically new and nonlinear approach for reconstructing the perturbated optical phase; while in the second, we show that oceanic dynamical information acquired at low resolution (pixel size : $22 \mathrm{kms}$ ) from altimetry can be transferred across the scales at high resolution sea surface temperature data (pixel size : $4 \mathrm{kms}$ ) to produce high resolution mapping of oceanic currents. In both the cases we use a proper wavelet decomposition tech- 
nique on the signal of the singularity exponents to help us inferring information along the scales of the signal.

The paper is organised as follows: in section 2 we present a brief discussion on the evolution of the theory of singularity exponents, in section 3 we present the numerical analysis for the singularity exponents and the idea of the most informative set within a signal. Theory behind the reconstruction of the whole signal from the most informative set is explained in section 4 . Notion of optimal wavelet, for inferring information pointwise in a cascade, is introduced in 5 . The experimental data used is discussed in section 6 and the results are shown in section 7 . Finally, we conclude in section 8.

\section{Universality class and multiscale organisation}

A power-law behaviour in the thermodynamical variables, and also time and spatial correlation functions, is commonly observed in systems with high order transitions. The underlying dynamics of such systems can be observed, at the macroscopic scale, in the form of a power-law [4]. It was soon realized that the exponents of the power laws define different classes: systems characterized by same values of singularity exponents belong to the same universality class, which implies the presence of a common macroscopic behaviour independent of the microscopic dynamics of each system [10]. Different singularity spectra of very different physical systems can match a same curve. Such a correspondance can be explained by the existence of a common underlying dynamical system, the universality class, responsible for similar statistical properties of information at macroscopic scale [10].

Previous works attempt to relate the general organisation of a multiscale structure with the existence of cascade process [4]. In these works, a multiscale signal $s$ is characterized by the power-law scaling in the order $p$ moments of some related variable $\mathbb{T}_{r}$, in the way:

$$
\left\langle\left|\mathbb{T}_{r} s\right|^{p}\right\rangle=A_{p} r^{\tau_{p}}+o\left(r^{\tau_{p}}\right) \quad(r \rightarrow 0)
$$

The existence of multplicative cascade process was first justified by Kolmogorov in his theory on turbulence [5]. Kolmogorov proposed the following: given two scales $r$ and $L, 0<r<L$, we can characterize the distribution of the velocity field by an injection parameter $\eta_{r / L}$ as:

$$
\left|\mathbb{T}_{r} s\right| \doteq \eta_{r / L}\left|\mathbb{T}_{L} s\right|
$$

where the symbol '''means that both sides are equally distributed and $\eta_{r / L}=$ $[r / L]^{\alpha}$. From this relation, the order $p$ moments have the following relation:

$$
\left\langle\left|\mathbb{T}_{r} s\right|^{p}\right\rangle=[r / L]^{\alpha p}\left\langle\left|\mathbb{T}_{L} s\right|^{p}\right\rangle
$$

Comparing equations (1) and (3) we get, $\tau_{p}=\alpha p$. However, experiments show that in the case of FDT, the relationship between $\tau_{p}$ and $p$ is not linear rather it 
is a convex bell-shaped curve, a condition konwn as 'anomalous scaling' [2]. To apply Kolmogorov's decomposition in anomalous scaling, certain assumptions have to be made:

- $\eta_{r / L}$ has to be interpreted as a random variable, independent of $L$.

- The variables $\eta_{r / L}$ has to be infinitely divisible to ensure downward process from scale $L$ to $r$ is verified directly or in several stages giving rise to the famous cascade process.

It has been verified [5] that an injection mechanism as the one proposed by Kolmogorov leads to the understanding of a underlying geometrical structure in a multiplicative cascade process, together with the knowledge of the exponents $\tau_{p}$ for infering information along the scales of the signal. This experimental outcome of self-similarity led researchers to propose a different model for the generation of exponents.

\section{Singularity Analysis in the Microcanonical Framework}

Criticality, and the associated critical exponents are key notions in Statistical Physics to understand phase transitions, which are prototypes of scale invariant phenomena [4]. The spectrum of singularity exponents in a system determine its multiscale properties which are accessible statistically. We will say that a signal $s$ is multiscale in a microcanonical sense, if for at least one multiscale functional dependant on scale $r$, it is assumed that for any point $\boldsymbol{x}$ the following equation holds:

$$
\mathbb{T}_{r} s(\boldsymbol{x})=\alpha(\boldsymbol{x}) r^{h(\boldsymbol{x})}+o\left(r^{h(\boldsymbol{x})}\right)(r \rightarrow 0)
$$

for some functions $\alpha(\boldsymbol{x})$ and $h(\boldsymbol{x})$. The exponent $h(\boldsymbol{x})$, which is a function of the point $\boldsymbol{x}$, is called a singularity exponent or Local predictability exponent (LPE) of the point [2]. The central problem is to compute at high numerical precision the value of $h(\boldsymbol{x})$ at point $\boldsymbol{x}$ : bad approximations of singularity exponents lead to poor reconstructions.

\subsection{Local Predictability Exponents}

According to microcanonical formulations [4], a multiscale signal is supposed to satisfy equation (4) for a family of functionals $\mathbb{T}_{r}$, at any point $\boldsymbol{x}$ in the signal domain, and have a singularity spectrum computed from singularity exponents as a convex curve function of $h$ [4]. Equation (4) is a pointwise and localized version of the definition used in introducing singularity spectrum [1,3]: we do not make use of statistical averages and grand ensembles as in practice such an ensemble average is not accessible. Rather, we seek to evaluate $h(\boldsymbol{x})$ at point $\boldsymbol{x}$. We denote $\mathcal{F}_{h}$ the component in the signal's domain associated to singularity exponent value $h$ as:

$$
\mathcal{F}_{h}=\{\boldsymbol{x}: h(\boldsymbol{x})=h\}
$$


In other words, we can say that each point $\boldsymbol{x}$ in the signal is characterized by a singularity exponent $h(\boldsymbol{x})$ which is typical to one component $\mathcal{F}_{h}$, i.e., the components are level sets of the function $h(\boldsymbol{x})$. This family of sets is naturally associated to the multiscale hierarchy in a signal and in the case of natural images, it is expected that there exists a particular set which comprises the point where sharp transitions within the signal are well recorded. We will call this set as the Most Singular Manifold or MSM. Geometrically speaking, it is the singularity component associated with the smallest possible value $h_{\infty}$, finite for signals corresponding to physical variables that cannot diverge. We will denote this set by $\mathcal{F}_{\infty}$ and can be expressesd as:

$$
\mathcal{F}_{\infty}=\left\{\boldsymbol{x}: h(\boldsymbol{x})=h_{\infty}=\min (h(\boldsymbol{x}))\right\}
$$

The MSM plays a fundamental role in the multiscale geometrical hierarchy of natural images. Visual inspection of this set reveals a structure which is characterized by the presence of 'edges' or contours in natural images [6]. It will be understood hereafter that the MSM contains the most informative set in an image so that the whole signal can be reconstructed from the restriction of its gradient to the MSM. Moreover we will see that singularity exponents lead to a notion of edge that matches much better across the scales than the edges computed from classical filtering methods. Before we go deeper into the subject of MSM and its application to reconstructible systems, we give a brief overview for the determination of the singualrity exponents.

\subsection{Singularity Analysis}

The singularity exponents for experimental, discretized data can be calculated using different methods [2], but for our case we will use the Unpredictable Points Manifold (herein referred to as UPM) [16]. According to this method, we make point estimates of the singularity exponent, namely:

$$
h(\boldsymbol{x})=\frac{\log \left(\tau_{\Psi} s\left(\boldsymbol{x}, r_{0}\right)\right) /\left\langle\tau_{\Psi} s\left(., r_{0}\right\rangle\right)}{\log r_{0}}+o\left(\frac{1}{\log r_{0}}\right)
$$

where $\left\langle\tau_{\Psi} s\left(., r_{0}\right\rangle\right.$ is the average value of the wavelet projection over the whole signal and $o\left(\frac{1}{\log r_{0}}\right)$ is a diminishing quantity and $r_{0}$ is the minimum scale. If the signal $s$ is an image of size $M \times N$, then we choose $r_{0}=1 / \sqrt{M \times N}$. The singularity exponents computed on our experimental dataset are shown in Fig 1.

The values $h(\boldsymbol{x})$ are computed for all points $\boldsymbol{x}$ within the signal domain. Now, coming back to MSM, sorting of these singularity exponent values based on a typical threshold value 0 defines the standard reconstruction set in the MSM method. Such a set often provides a robust and accurate reconstruction and is defined by:

$$
\mathcal{E}_{\mathrm{MSM}}=\bigcup_{h_{\infty} \leq h \leq 0} \mathcal{F}_{h}
$$



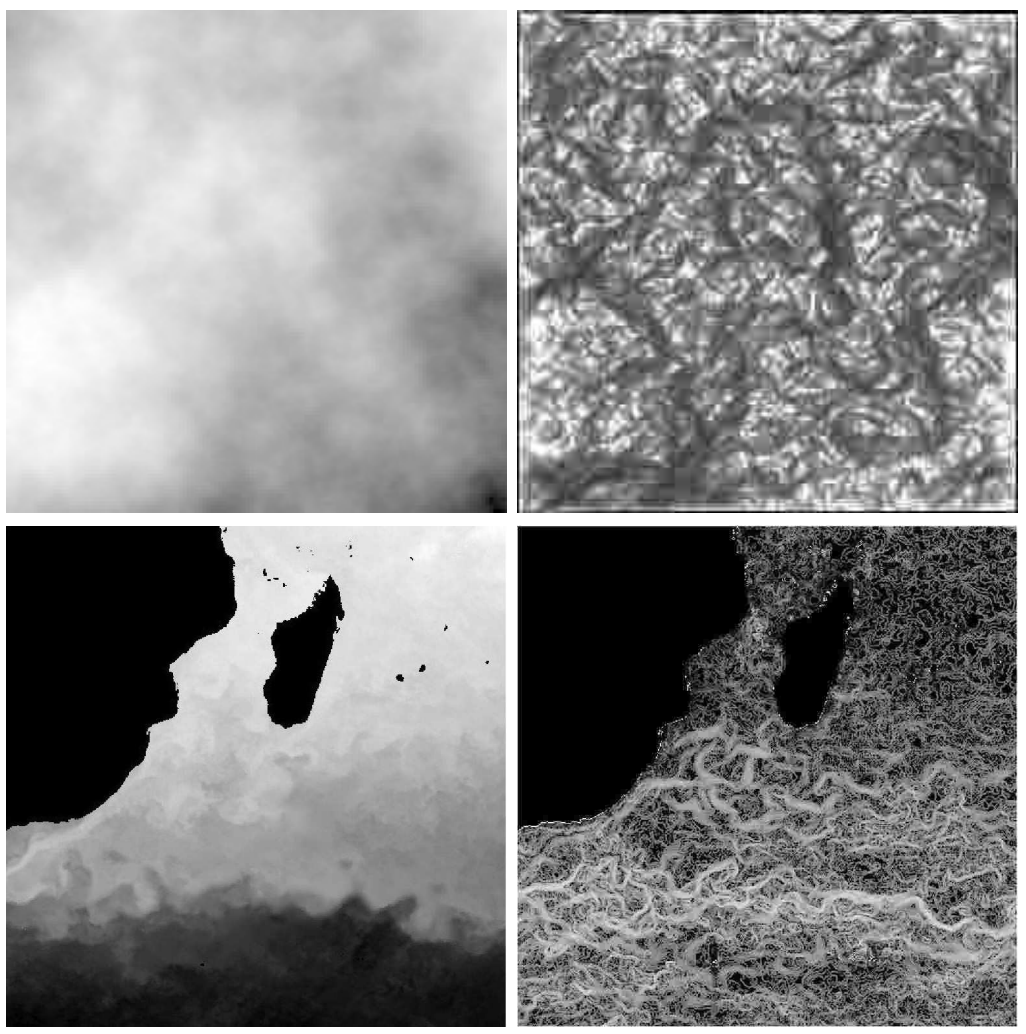

Fig. 1: Top left: Image of a simulated optical phase perturbated by atmospheric turbulence. The image corresponds to a $128 \times 128$ pixels sub-image extracted from an original $256 \times 256$ pixels image to avoid the pupil boundary. Top right: Image of the singularity exponents computed from the phase data. Bottom left: Excerpt of the Agulhas current below the coast of South Africa (sea surface temperature image: each pixel record the temperature of the upper layer of the sea). Bottom right: Singularity exponents of the Agulhas current.

\section{Reconstructible Systems}

In this section, we are led to find mathematically a functional $\mathcal{G}$ which permits the reconstruction of the signal's gradient from its restriction to the MSM. The functional must satisfy the properties of being deterministic, linear, translationallyinvariant, isotropic and yield correct power spectrum of natural images. We consider the gradient measure of the signal $s=\nabla s(\boldsymbol{x})$ and integrate it over the multifractal set of most unpredictable points $\mathcal{F}_{\infty}$. A deterministic representation of the gradient measure for the signal can be:

$$
\nabla s(\boldsymbol{x})=\mathcal{G}\left(\left.\nabla s\right|_{\mathcal{F}_{\infty}}\right)(\boldsymbol{x})
$$


Considering the fact of $\mathcal{G}$ being linear, an integral representation can be given by:

$$
\nabla s(\boldsymbol{x})=\int_{\mathcal{F}_{\infty}} \nabla s(\boldsymbol{y}) G(\boldsymbol{x}, \boldsymbol{y}) \mathrm{d} \boldsymbol{y}
$$

where $G(\boldsymbol{x}, \boldsymbol{y})$ is a density measure of the function $\mathcal{G}$ and is a $2 \times 2$ matrix. Using isotropy, standard power spectrum (in the form $1 /\|\boldsymbol{f}\|^{2}$ ) for the associated spectral measures of natural images, one obtains the following formula $[4,6]$ expressed in Fourier space:

$$
\hat{s}(\boldsymbol{f})=\frac{\boldsymbol{f} . \widehat{\left.\nabla s\right|_{\mathcal{F}_{\infty}}}(\boldsymbol{f})}{i\|\boldsymbol{f}\|^{2}}
$$

where $i$ is the imaginary unit, $i \equiv \sqrt{-1}$ and ^ denotes the Fourier transform. We normalize the result by taking the vector field $\boldsymbol{v}_{\mathbf{0}}$ unitary and normal to the MSM instead of $\left.\nabla s\right|_{\mathcal{F}_{\infty}}$; where $\boldsymbol{v}_{\mathbf{0}}(\boldsymbol{x})=\nabla s(\boldsymbol{x}) \delta_{\mathcal{F}_{\infty}}, \delta_{\mathcal{F}_{\infty}}$ being the density of the proper Hausdorff measure restricted to the set $\mathcal{F}_{\infty}[6]$. We therefore perform integration over all the space (the restriction is still present, but now introduced by $\left.\boldsymbol{v}_{\mathbf{0}}\right)$ :

$$
\hat{s}(\boldsymbol{f})=\frac{\boldsymbol{f} \cdot \widehat{\left.\boldsymbol{v}_{\mathbf{0}}\right|_{\mathcal{E}}}(\boldsymbol{f})}{i\|\boldsymbol{f}\|^{2}}
$$

Fourier inversion of this formula gives the reconstruction of the image from the restriction of the gradient field to the MSM. Results of reconstruction on the MSM of experimental datasets, and their performance over classical edge detection algorithms, are shown in tables 2 and 1. It is seen that in the case of acquisitions of turbulent signals, the reconstruction based on the MSM (we call it MSM in tables 2 and 1) performs significantly better among the algorithms tested. In fact, when it comes to the case of turbulent signals, the classical edge detectors like Sobel [20], Prewitt [18], Roberts [17], Laplacian of Gaussian (LoG) [19,21], Zerocross [22, 24], Canny [23] to a more recent non-linear approach called NLFS [7], dedicated to the computation of edges in digital images, are systematically outperformed by MSM in terms of reconstruction from a compact representation of its edge pixels. As a consequence, the fundamental notion of edge, which is a basic multiscale feature, is much more well encoded by the set $\mathcal{E}_{\mathrm{MSM}}$ defined in equation (8). This tends to show that singularity exponents are good candidates for an accurate multiresolution analysis. In the next section, we develop the notion of optimal wavelet.

\section{Inferring Information across the scales using microcanonical analysis}

To infer the cascading properties pointwise (called microcanonical cascade) we introduce the concept of optimal wavelet. Let $s(\boldsymbol{x})$ be a multiscale signal and let $\Psi(\boldsymbol{x})$ be a wavelet. We define the wavelet projection of $s$ on $\Psi$ at position $\boldsymbol{x}$ and resolution $r$ as:

$$
T_{\Psi}|\nabla s|(\boldsymbol{x}, r)=\int|\nabla s|(\boldsymbol{y}) \Psi\left(\frac{\boldsymbol{x}-\boldsymbol{y}}{r}\right) d \boldsymbol{y}
$$


We can now define a random variable $\zeta_{r / L}(\boldsymbol{x})$ as

$$
T_{\Psi}|\nabla s|(\boldsymbol{x}, r)=\zeta_{r / L}(\boldsymbol{x}) T_{\Psi}|\nabla s|(\boldsymbol{x}, L)
$$

Now, we can talk about a wavelet $\Psi$ which, if determined, will make $\zeta_{r / L}(\boldsymbol{x})$ independent of $T_{\Psi}|\nabla s|(\boldsymbol{x}, L)$. Such a wavelet is called an optimal wavelet. In subsection Optimal Wavelet Analysis, we propose a new algorithm for a very robust detection of the optimal wavelet in $2 \mathrm{D}$ signals. The new methodology helps us to detect the presence of an optimal wavelet, in a totally unconstrained way, from the signal itself. Once determined, the optimal wavelet has the potential of unlocking the signal's microcanonical cascading properties through simple wavelet multiresolution analysis [8].

\subsection{Multiresolution Analysis \& fast wavelet transform}

Multiresolution analysis is a mathematical formalism that deals with the phenomenon of detail-structured viewing of objects [8]. Data redundancy is minimized by use of dyadic wavelet sequences which are hilbertian frames associated to dyadic partition of the space/frequency domain.

Any signal $|s\rangle$ can be represented in a dyadic wavelet basis of mother wavelet $|\Psi\rangle[13]$ as follows (from now on we use the notation $|s\rangle$ for the signal):

$$
|s\rangle=\sum_{j=-\infty}^{\infty} \sum_{k} \alpha_{j, k}\left|\Psi_{j, k}\right\rangle
$$

where

$$
\left|\Psi_{j, k}\right\rangle(\boldsymbol{x})=2^{j / 2} \Psi\left(2^{j} \boldsymbol{x}-k\right)
$$

and $\alpha_{j, k}$, are called wavelet coefficients. The wavelet coefficients $\alpha_{j, k}$ can be obtained by a simple projection of the signal $|s\rangle$ onto the basis function $\Psi_{j, k}$, namely:

$$
\alpha_{j, k}=\left\langle s \mid \Psi_{j, k}\right\rangle
$$

The decomposition process using multiresolution analysis gives rise to an image fourth smaller than the previous one. Therefore, each parent coefficient $\alpha_{p} \equiv \alpha_{j-1,[k / 2]}$, at the coarser scale $j-1$, covers the same spatial extent of four children coefficients $\alpha_{c} \equiv \alpha_{j, k}$ at the finer scale $j$.

\subsection{Approximation of microcanonical cascade}

In the wavelet analysis of $2 \mathrm{D}$ signals, persistence along the scales implies a realtion of the form between the wavelet coefficients:

$$
\alpha_{c}=\eta_{1} \alpha_{p}+\eta_{2}
$$

with $\eta_{1}, \eta_{2}$ : random variables independant of $\alpha_{c}$ and $\alpha_{p}$ and also independant of each other. For an optimal wavelet the above equation takes the form $\alpha_{c}=\eta_{1} \alpha_{p}$. 


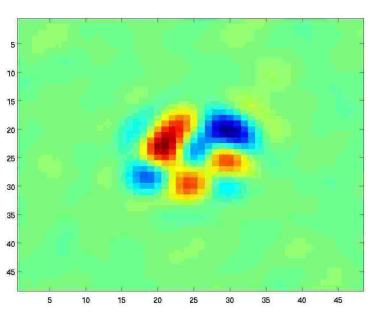

(a)

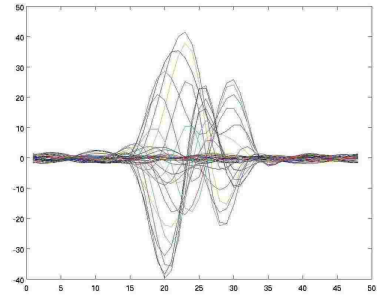

(b)

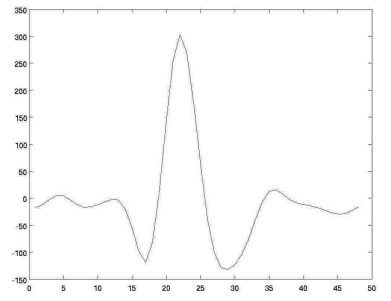

(c)

Fig. 2: (a) Sub-image at $48 \times 48$ pixels resolution obtained after orientation with the sign with the MCE (b) 2-D plot of the sub-image (c) 2-D plot for the sum over the columns.

\subsection{Optimal Wavelet Analysis}

Any given signal $|s\rangle$ can be represented in terms of their cascade variables $\eta$ and wavelet coefficients $\alpha$ as:

$$
|s\rangle=\sum_{j \neq 0, k} \prod_{j^{\prime}, k} \eta_{j^{\prime},\left[k / 2^{\left.j-j^{\prime}\right]}\right.} \alpha_{0,0}\left|\Phi_{j, k}\right\rangle+\alpha_{0,0}\left|\Phi_{0,0}\right\rangle
$$

where $\Phi_{j, k}$ is the wavelet basis for the optimal wavelet. Experimental observations show that the expectation of the signal $\langle\mid s\rangle\rangle=0$ as $\left\langle\alpha_{0,0}\right\rangle=0$ due to symmetry.

We multiply the sign of $\alpha_{0,0}$ i,e, $\sigma_{0,0}$ with the signal $s$ and then compute the average. Since, in our case we have an ensemble of dynamically equivalent signals, we compute the average over $\left|s^{i}\right\rangle$ to get the expected value for all these signals; where $i$ is the index of an ordering of the signals. Equation (19) can be generalized to:

$$
\left.\left\langle\sigma_{0,0}^{i} \mid s^{i}\right\rangle\right\rangle=\left\langle\left|\alpha_{0,0}\right|\right\rangle\left|\Phi_{0,0}\right\rangle
$$

We try to estimate the sign of $\alpha_{0,0}$. Let $\epsilon_{0,0}$ be the estimation, we then have

$$
\left.\left\langle\epsilon_{0,0}^{i} \mid s^{i}\right\rangle\right\rangle=\left\langle\epsilon_{0,0} \sigma_{0,0}\left|\alpha_{0,0}\right|\right\rangle\left|\Phi_{0,0}\right\rangle \propto \Phi_{0,0}
$$

Principle application of finding the optimal wavelet on a given set of images is quite simple. The procedure is as follows:

- We subdivide a given image $\left|s^{i}\right\rangle$ over small areas of equal sizes and normalize individually every sub-image.

- We find the correlation between the sub-images of $\left|s^{i}\right\rangle: C_{i}=\left\langle s^{i} \mid s^{k}\right\rangle$.

- For every $i$ we find the average of the correlation.

- We then find for which $l$, the average correlation is maximum. Let it be $i^{*}$.

- We call $\left|s^{i^{*}}\right\rangle$ as the most central element (MCE). 
Since we don't know $\Phi_{0,0}$, we make the wavelet projection of the signal on the element which has the most dependancy with all the other elements (dominant presence of the term $\left.\left\langle\left|\alpha_{0,0}\right|\right\rangle\left|\Phi_{0,0}\right\rangle\right)$; i,e, the MCE. So, we have

$$
\begin{aligned}
\epsilon_{0,0}^{i} & =\sigma\left(C_{i, i *}\right)=\sigma\left(\left\langle s^{i} \mid s^{i *}\right\rangle\right) \\
& =\left|\alpha_{0,0}^{*}\right| \sigma_{0,0} \sigma_{0,0}^{*}\left\langle s \mid \Phi_{0,0}\right\rangle
\end{aligned}
$$

If we have a correct estimate of the sign, we can say $\left.\left.\left\langle\epsilon_{0,0}^{i} \mid s^{i}\right\rangle\right\rangle \propto\left\langle\sigma_{0,0}^{i} \mid s^{i}\right\rangle\right\rangle$. And we estimate the wavelet from equation (21). Since wavelets are normalized by definition, we can cancel the proportionality factor in equation (21):

$$
\left.\left.\left.\left\langle\epsilon_{0,0}^{i} \mid s^{i}\right\rangle\right\rangle=\left\langle\sigma\left(C_{i, i *}\right) \mid s^{i}\right\rangle\right\rangle=\left\langle\sigma\left(\left\langle s^{i} \mid s^{i *}\right\rangle\right) \mid s^{i}\right\rangle\right\rangle
$$

We have tested this algorithm on Benzi model [9] to construct multiaffine fields based on an order 2 gaussian wavelet decomposition. The preliminary results are shown in Fig 2. Since, the process of finding an optimal wavelet is still under review and subjected to constant experimentation, we approximate the optimal wavelet by a Battle-Lemarié wavelet which is found to give an acceptable approximation of the optimal wavelet.

\section{DATA}

\subsection{Atmospheric Phase}

The data is shown in Fig 1 (top). The datasets consists in simulated optical phase perturbated by the Earth's upper layer turbulent atmosphere. The optical phase corresponds to the acquisition of a point source (representing a star far away enough in outer space so that the optical phase reaching the telescope is in the form of planar wavefronts). These data are provided by the French Aerospace Lab-ONERA, and they have the following imaging characteristics:

- diameter of the telescope: $8 \mathrm{~m}$,

- seeing at 5 microns: 0.85 arcseconds,

- wind's speed: $12.5 \mathrm{~m} / \mathrm{s}$,

- acquisition frequency: $250 \mathrm{~Hz}$,

- pupil size: $256 \times 256$ pixels, but for our experimental purpose we take $128 \times 128$ pixels from the centre to eliminate boundary effects.

We have the Hartmann-Shack (HS) acquisition of the $x$ and $y$ slopes for the phase data provided by onera given by 208 effective HS sub-pupils (size $16 \times 16$ ) which samples the pupil of the telescope. The distribution of the sub-pupils within the telescope is shown in Fig 4(a). Fig 4(b) and Fig 4(c) shows the $x$ and $y$ low resolution acquisition of the phase data, which gives us an approximation of low resolution $x$ and $y$ components of the gradients for the phase, by the HS sensor. 


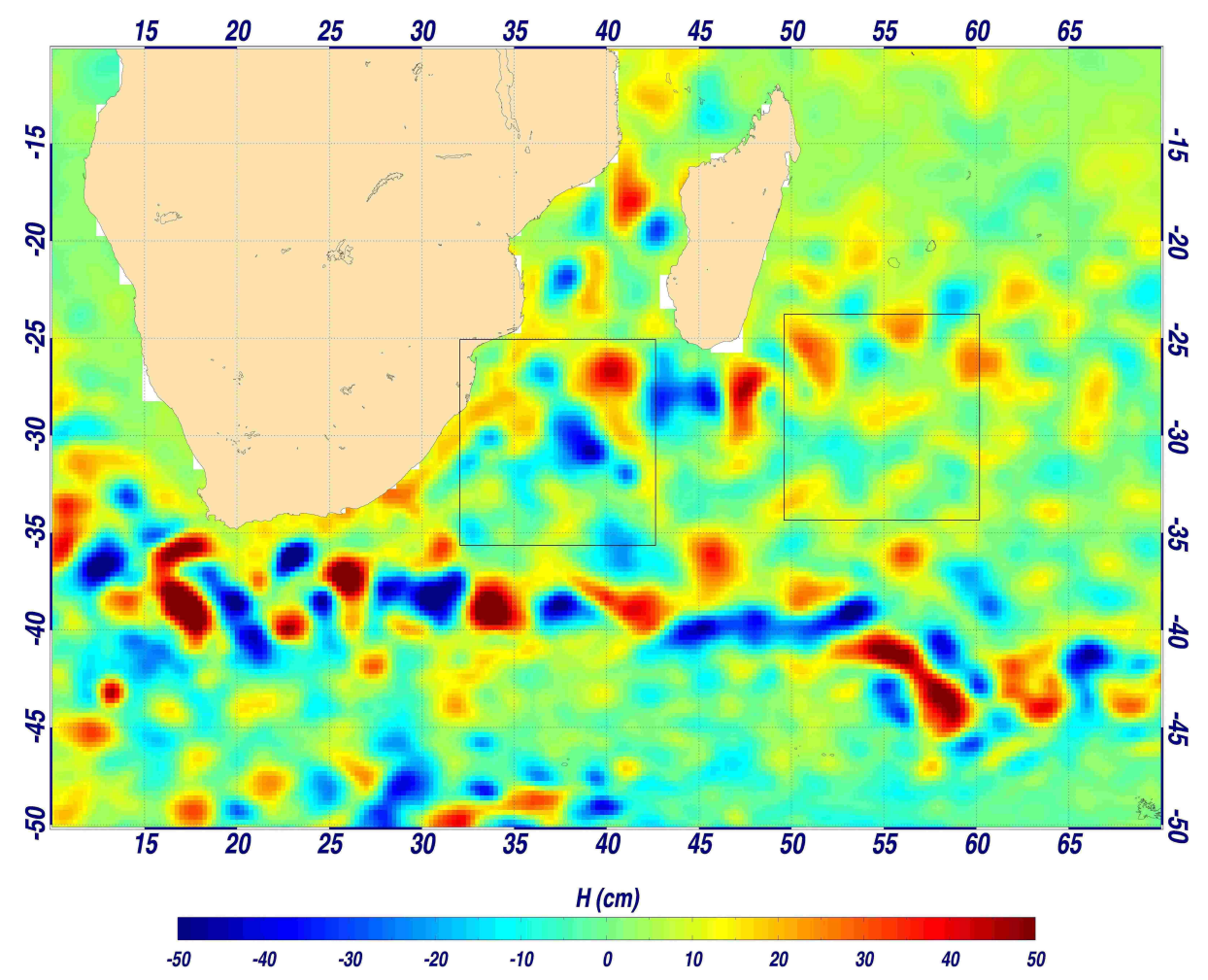

(a)

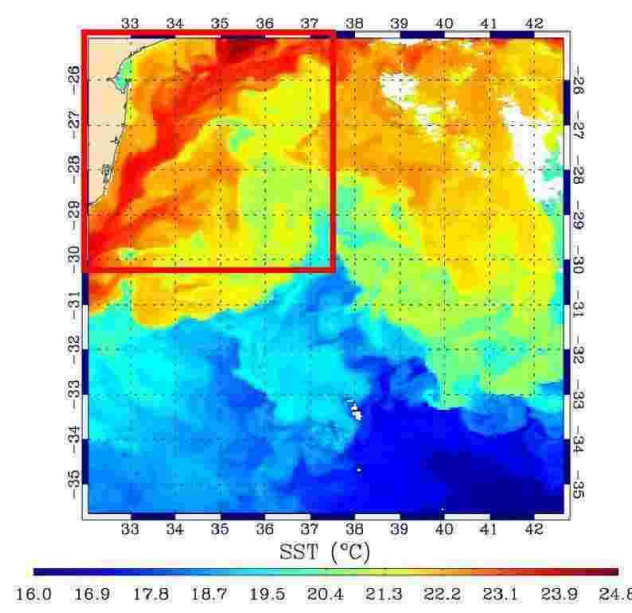

(b)

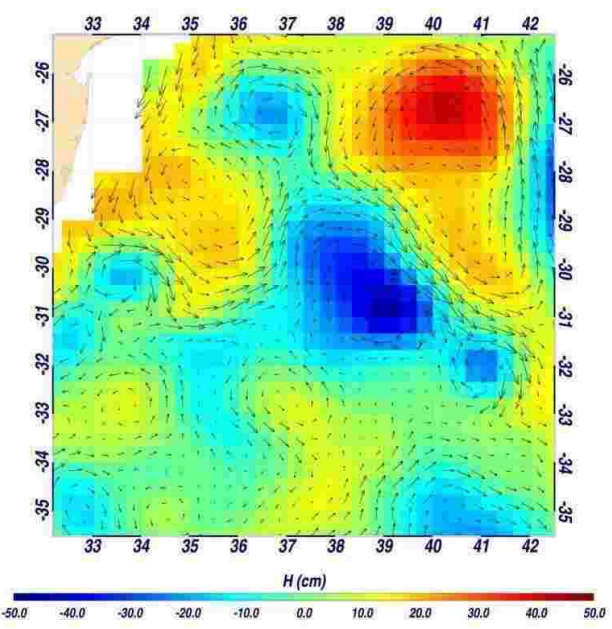

(c)

Fig. 3: (a) Altimetry data (b) Sea Surface Temperature (SST) acquired by MODIS satellite on August 2, 2007 (c) low-resolution motion field derived from altimetry. 


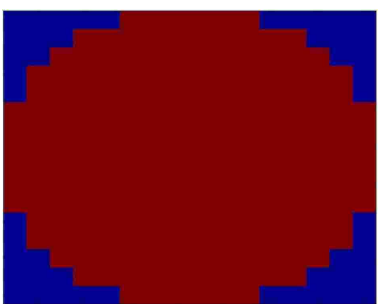

(a)

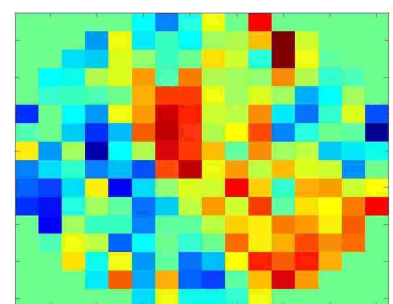

(b)

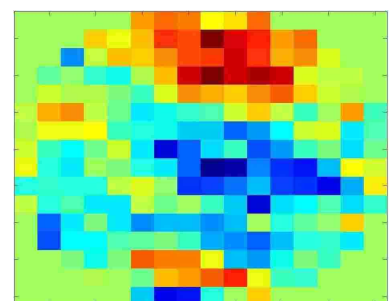

(c)

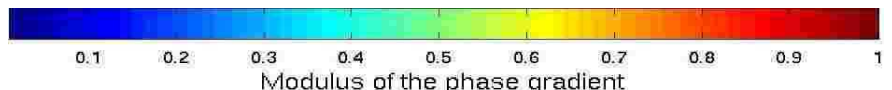

Fig. 4: (a) Distribution of the sub-pupils within the telescope (b) HS acquisition of the $x$ slope for the phase data (c) HS acquisition of the $y$ slope for the phase data.

\subsection{Sea Surface Temperature}

Sea surface temperature data (SST) are global acquisitions of the temperature of the ocean's upper layer. Data is radiometrically corrected so that pixels values represent celsius degrees. Data is acquired by the MODIS instrument orbiting around earth, pixel resolution is $4 \mathrm{kms}$, data is acquired on 02 August 2007. In our experiment, we also use low resolution products representing geostrophy and Ekman currents deduced from altimetry data according to method exposed in [26]. Pixel size of altimetry products is $22 \mathrm{kms}$. Figure 3(a) shows the altimetry, figure 3(b) the MODIS data and figure 3(c) shows the low-resolution motion field derived from altimetry according to [26].

\section{Results}

\subsection{Edge detection and singularity exponents}

First we detail the comparison results on edge detection using classical linear filtering and the set provided by equation (8). Reconstruction has been performed on the edge files computed on the phase and sea surface temperature images. Performance of the reconstruction on classical edge detectors to a more recent nonlinear derivative approach (called NLFS) [7] and MSM has been presented in table 1 . Also, we evaluate the quality of the reconstruction using the peak signal to noise ratio (psnr, expressed in decibels $\mathrm{dB}$ ) defined by:

$$
\mathrm{psnr}=-20.0 \times \log _{10}\left(\frac{1}{\lambda(\Omega)} \frac{\left(\int_{\Omega}\left(s(\boldsymbol{x})-s_{r}(\boldsymbol{x})\right)^{2} \mathrm{~d} \boldsymbol{x}\right)^{1 / 2}}{\Delta_{s}}\right)
$$

where $\Omega$ is the image domain, $\lambda(\Omega)$ its Lebesgue measure (image size), $s$ the original image, $s_{r}$ the reconstructed image, and $\Delta_{s}$ is the dynamical range of $s$, 
i.e. the difference between the maximal and minimal values. Better reconstructions tend to have a higher psnr. A quantitative evaluation of the results are presented in table 2 .

\subsection{Reconstruction of optical phase}

Also, we show that the application of wavelet multiresolution analysis technique on the signal (optical phase perturbated by atmospheric turbulence) of the singularity exponents provided by a simple approximation of an optimal wavelet (here a third order Battle-Lemarié wavelet) help us to infer information along the scales of the signal which in turn can be used to properly reconstruct the signal from low resolution to high resolution. The process is summarized below:

- We first compute the third order Battle-Lemarié wavelet coefficients associated to the signal of the singularity exponents computed on the perturbated phase signal,

- for each component $(x$ and $y)$ of the phase gradient at low reslution $(16 \times 16$ sub-image, see section 6.1), back project the component from low to high resolution to get a phase's gradient at higher spatial resolution of $128 \times 128$.

Consequently, we reconstruct the phase by performing inverse gradient operation on the norms of the gradients. We also check the robustness of our reconstruction algorithm by adding different proportions of gaussian white noise to the data. Results obtained, as shown in Fig 6, show visual resemblance of the reconstructed signal with the original one.

\subsection{High resolution ocean dynamics}

In this experiment, the low resolution vector field shown in figure 3(c) and derived from altimetry data is used to generate a high resolution vector field corresponding to SST data. First, the singularity exponents are computed on SST data. Then a multiresolution analysis is performed on the resulting singularity exponents from SST spatial resolution $(4 \mathrm{kms})$ down to altimetry resolution (22 $\mathrm{kms})$. The low resolution vector field shown in figure 3(c) is propagated, componentwise, up to SST resolution and the resulting vector field is prefiltered using an $1 /\|f\|$ filter. The results are shown in figure 5: a high resolution vector field representing the ocean dynamics at resolution $4 \mathrm{kms}$ is obtained from the multiresolution analysis of the singularity exponents. Validation has been performed on the outputs of a 3D simulation model, and shows proper reconstruction of the high resolution vector field both in norm and direction: $80 \%$ of vectors are correctly computed. This method provides a very interesting alternative to classical motion computation techniques that use conservation hypothesis (optical flow) or Maximum Correlation methods. 
Table 1: Performance of different edge detection algorithms. row 1: Atmospheric phase data. row 2: SST data of the Agulhas current below the coast of South Africa.

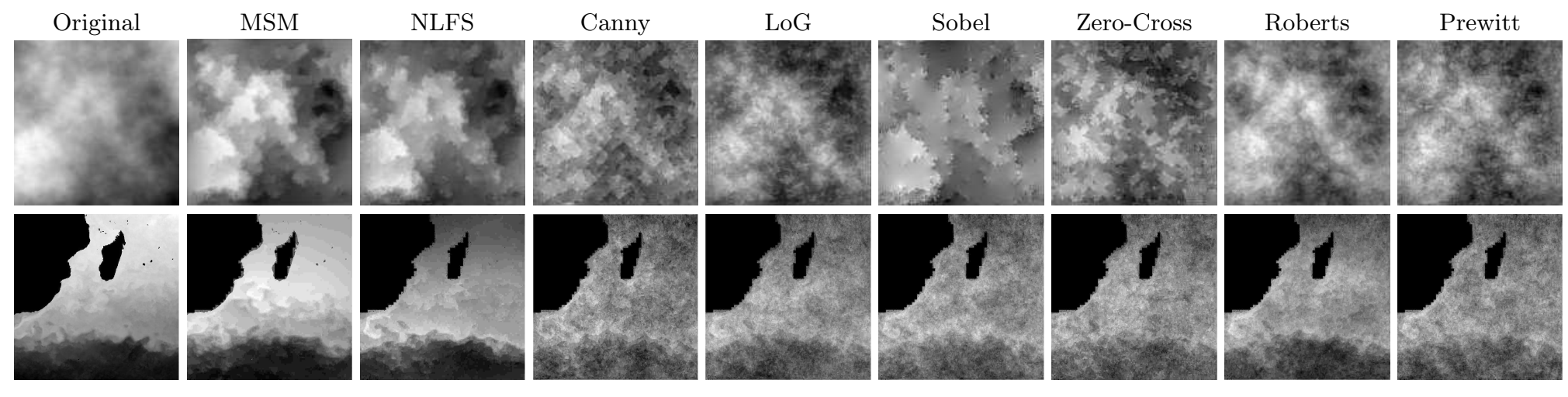

Table 2: Evaluation of edge detection algorithms

\begin{tabular}{|c|c|c|c|c|}
\hline Image & Algorithm & Parameter(s) & Density & $\begin{array}{l}\text { Reconstruction } \\
\text { (psnr) }\end{array}$ \\
\hline sea temp. & NLFS [7] & $\sigma=0.2$ & $22.24 \%$ & $10.15 \mathrm{~dB}$ \\
\hline sea temp. & Canny & $\sigma=0.001, \alpha=01$ & $13.094 \%$ & $9.65 \mathrm{~dB}$ \\
\hline sea temp. & Laplacian & $\sigma=0.001, \alpha=01$ & $24.47 \%$ & $10.16 \mathrm{~dB}$ \\
\hline sea temp. & Sobel & $\sigma=0.001$ & $24.58 \%$ & $9.58 \mathrm{~dB}$ \\
\hline sea temp. & Zero-crossing & $\sigma=0.001$ & $13.95 \%$ & $9.60 \mathrm{~dB}$ \\
\hline sea temp. & Roberts & $\sigma=0.001$ & $27.97 \%$ & $10.22 \mathrm{~dB}$ \\
\hline sea temp. & Prewitt & $\sigma=0.001$ & $24.83 \%$ & $9.83 \mathrm{~dB}$ \\
\hline sea temp. & MSM & parameter free & $17.24 \%$ & $11.30 \mathrm{~dB}$ \\
\hline phase & NLFS [7] & $\sigma=0.25$ & $24.92 \%$ & $8.30 \mathrm{~dB}$ \\
\hline phase & Canny & $\sigma=0.001, \alpha=01$ & $14.11 \%$ & $7.25 \mathrm{~dB}$ \\
\hline phase & Laplacian & $\sigma=0.001, \alpha=01$ & $28.48 \%$ & $7.24 \mathrm{~dB}$ \\
\hline phase & Sobel & $\sigma=0.3$ & $5.83 \%$ & $6.48 \mathrm{~dB}$ \\
\hline phase & Zero-crossing & $\sigma=0.001$ & $15.88 \%$ & $6.61 \mathrm{~dB}$ \\
\hline phase & Roberts & $\sigma=0.001$ & $34.74 \%$ & $7.77 \mathrm{~dB}$ \\
\hline phase & Prewitt & $\sigma=0.001$ & $26.72 \%$ & $6.96 \mathrm{~dB}$ \\
\hline phase & MSM & parameter free & $15.75 \%$ & $13.18 \mathrm{~dB}$ \\
\hline
\end{tabular}



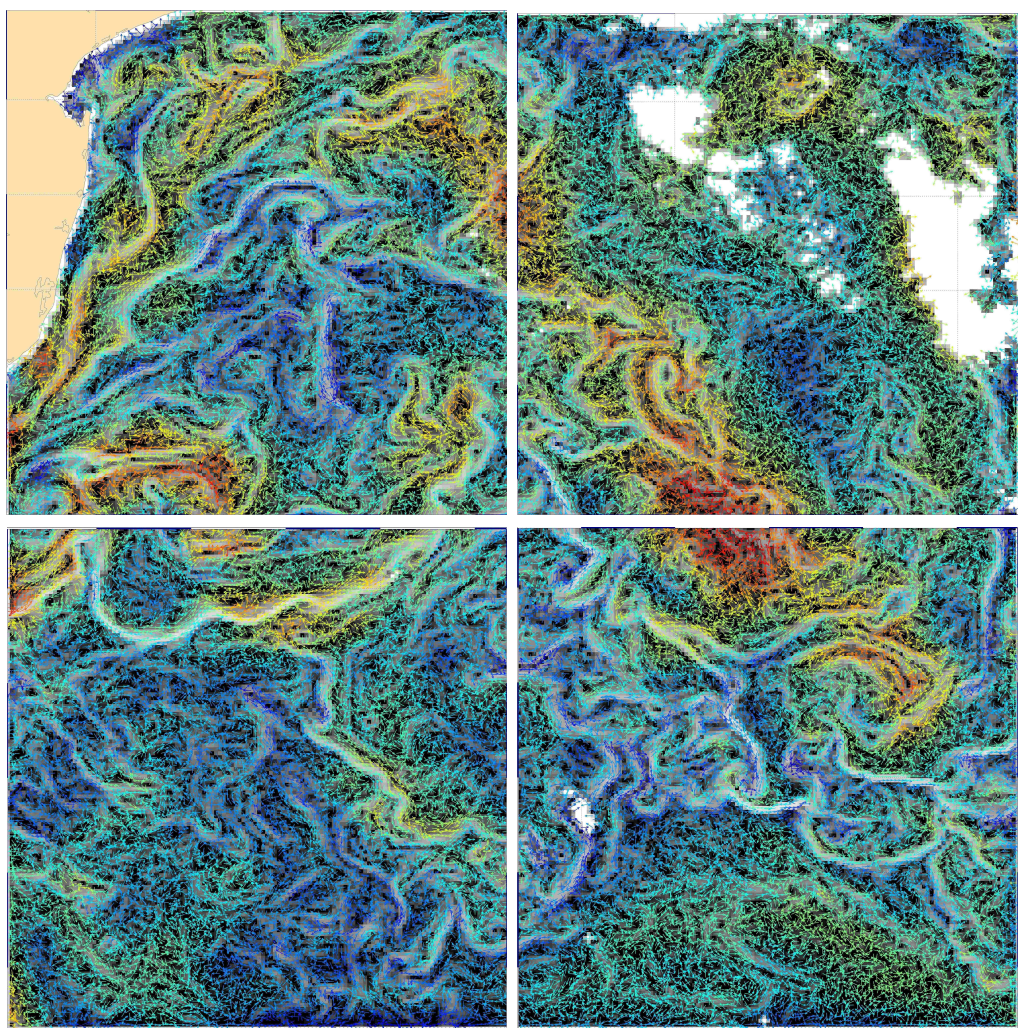

Singularity Exponents

\begin{tabular}{lllllllllll}
\hline 0.60 & -0.48 & -0.36 & -0.24 & -0.12 & 0.00 & 0.12 & 0.24 & 0.36 & 0.48 & 0.60
\end{tabular}

Fig. 5: Vector field computed at high resolution SST MODIS data using the low resolution altimetry of Fig 3(c) and the multiresolution analysis of the SST singularity exponents as explained in section 7 . The color of the vectors indicate their norm from $0.0 \mathrm{~cm} \cdot \mathrm{s}^{-1}$ (blue) to $83.9 \mathrm{~cm} \cdot \mathrm{s}^{-1}$ (red). In the background we also display the singularity exponents.

\section{Conclusion}

In this work we set up and study a multiresolution analysis scheme general enough to suit the case of acquisitions of general complex systems. We first study geometrically localized singularity exponents in natural signals, computed in a microcanonical framework, from which singularity spectra can be derived. We study their relations with high order transitions in associated phase spaces, and conclude that they unlock a notion of transition that outperforms all classical «linear filtering» approaches for edge detection in the case of 2D images. Edges 


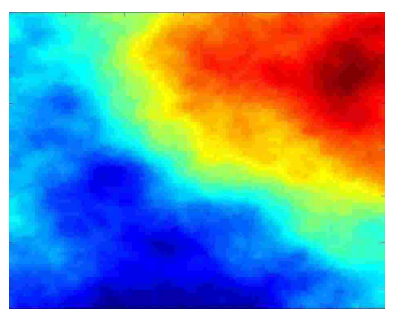

(a)

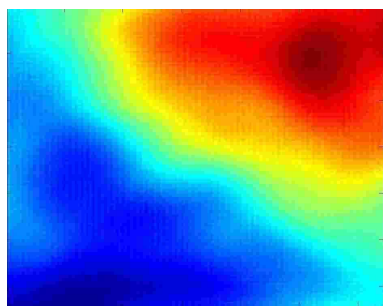

(d)

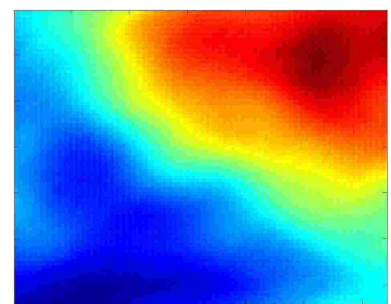

(b)

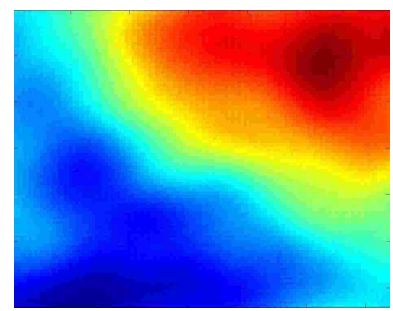

(e)

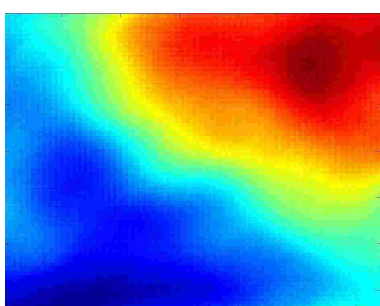

(c)

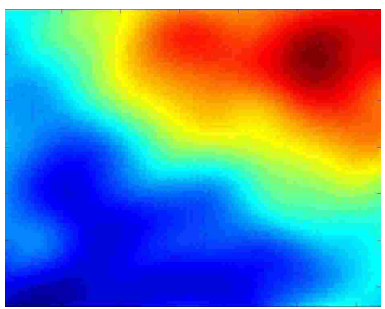

(f)

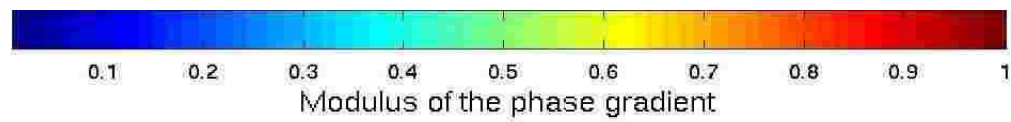

Fig. 6: (a) The original phase. Reconstructed phase (b) without noise. (c) with an input SNR of $40 \mathrm{~dB}$. (d) with an input SNR of $26 \mathrm{~dB}$. (e) with an input SNR of $14 \mathrm{~dB}$. (f) with an input SNR of $6 \mathrm{~dB}$.

are typical multiscale features, which should maximize information content in natural signals. We study the performance of reconstructible systems both with transitions associated to singularity exponents and the edge pixels provided by standard edge detection techniques. Examples are chosen among the most difficult natural signals: acquisition of turbulent phenomena (perturbated optical phase and ocean dynamics acquired from space). We study a multiresolution analysis scheme associated to the signal of singularity exponents, and in doing so we provide an effective determination of optimal wavelets, which are wavelets whose associated multiresolution analysis is optimal w.r.t inference across the scales. We show the power of the approach by studying two specific examples: the reconstruction of the phase perturbated by atmospheric turbulence applied to adaptive optics and the generation of high resolution ocean dynamics from low resolution acquired altimetry signals. The method is general enough to provide an effective approach to infer sub-pixel information in most natural complex signals. 


\section{Acknowledgment}

Suman Kumar Maji's PhD is funded by a CORDIS grant and Région Aquitaine OPTAD research project grant.

\section{References}

1. O. Pont, A. Turiel, C. Perez-Vicente: Empirical evidences of a common multifractal signature in economic, biological and physical systems. Physica A, Elsevier, 388, 2009, 2025-2035.

2. A. Turiel, C. Perez-Vicente, J. Grazzini: Numerical methods for the estimation of multifractal singularity spectra on sampled data: A comparative study. Journal of Computational Physics, Elsevier, 216, 2006, 362-390.

3. A. Arneodo, E. Bacry, J. Muzy: The thermodynamics of fractals revisited with wavelets. Physica A 213, 232-275, 1995.

4. A. Turiel, H. Yahia and C. Perez-Vicente: Microcanonical Multifractal Formalism: a geometrical approach to multifractal systems. Part I: singularity analysis. Journal of Physics A: Mathematical and Theoretical, vol 41, doi: 10.1088/1751$8113 / 41 / 1 / 015501$.

5. U. Frisch: 1995 Turbulence (Cambridge: Cambridge University Press).

6. A. Turiel and A. del. Pozo Reconstructing images from their most singular fractal manifold. IEEE Transactions Image Processing, 2002, 11, 345-50.

7. O. Laligant, F. Truchetet: A nonlinear derivative scheme applied to edge detection. IEEE PAMI, 2010, 32, 242-257.

8. C. Pottier, A. Turiel, V. Garçon: Inferring missing data in satellite chlorophyll maps using turbulent cascading. Remote Sensing of Environment, Elsevier, 112, 2008, 4242-4260.

9. R. Benzi, L. Biferale, A. Crisanti, G. Paladin, M. Vergassola, A. Vulpiani: A random process for the construction of multiaffine fields. Physica D: Nonlinear Phenomena, Volume 65 Issue 4, June 15, 1993.

10. S. Lovejoy, D. Schertzer: Multifractals, universality classes, satellite and radar measurements of clouds and rain. Journal of Geophysical Research 95, 2021-2034, 1990.

11. G. Parisi, U. Frisch: On the singularity structure of fully developed turbulence Turbulence and Predictability in Geophysical Fluid Dynamics. Proc. Intl School of Physics E. Fermi ed M Ghil, R Benzi and G Parisi (Amsterdam: North-Holland) pp 84-7, 1985.

12. A. Turiel and N. Parga: The multi-fractal structure of contrast changes in natural images: from sharp edges to textures. Neural Computation 12, 763-93, 2000.

13. Stéphane Mallat, A Wavelet Tour of Signal Processing, Academic Press, 2nd Edition, 1999.

14. G. Boffetta, M. Cencini, M. Falcioni, et al: Predictability: a way to characterize complexity. Physics Reports 356 (6): 367-474 JAN 2002.

15. O. Pont, A. Turiel, C. Perez-Vicente, Description, modelling and forecasting of data with optimal wavelets, J.Econ Interact Coord, 2009, 4: 39-54, doi 10.1007/s11403009-0046-x.

16. O. Pont, A. Turiel, H. Yahia: An Optimized Algorithm for the Evaluation of Local Singularity Exponents in Digital Signals. IWCIA, 6636, 2011, 346-357. 
17. L. G. Roberts: Machine perception of three dimensional solids. in Optical and Electro-Optical Information Processing, J. T. Tippett et al., Eds. Cambridge, MA: MIT Press, 1965.

18. J. Prewitt: Object Enhancement and Extraction. Picture Process Psychopict, 1970, pp. 75-149.

19. A. Rosenfeld: Picture Processing by Computer. New York: Academic Press, 1969.

20. I. Sobel: Neighbourhood coding of binary images fast contour following and general array binary processing. Computer Graphics and Image Processing 8, pp. 127-135, 1978.

21. D. Marr, E. Hildreth: Theory of edge detection. Proc. Royal Soc. London B 207, 187-217, 1980.

22. R. M. Haralick: Digital step edges from zero crossing of second directional derivatives. IEEE PAMI 6, 58-68, 1984.

23. J. Canny: A computational approach to edge detection. IEEE PAMI 8, 679-698, 1986.

24. V. Torre, T. A. Poggio: On edge detection. IEEE PAMI 8, 147-163, 1986.

25. O. Faugeras: Three-dimensional computer vision:a geometric viewpoint. MIT Press 1993, ISBN: 0-262-06158-9.

26. J. Sudre and R. Morrow: Global surface currents, a high resolution product for investigating ocean dynamics, 58, 101-118, doi:10.1007/s10236-008-0134-9, 2008. 